Editorial

\title{
Neural Systems in Distributed Computing and Artificial Intelligence
}

This Neurocomputing special issue presents the post-proceedings of the International Conference on Practical Applications on Agents and Multi-Agent Systems (PAAMS 2015) held in Salamanca in June 3th-5th, 2015. PAAMS provides an international forum to present and discuss the latest scientific developments and their effective applications, to assess the impact of the approach, and to facilitate technology transfer. PAAMS started as a local initiative, but has since grown to become the international yearly platform to present, to discuss, and to disseminate the latest developments and the most important outcomes related to real-world applications. It provides a unique opportunity to bring multi-disciplinary experts, academics and practitioners together to exchange their experience in the development and deployment of Agents and Multi-Agent Systems. PAAMS intends to bring together researchers and developers from industry and the academic world to report on the latest scientific and technical advances on the application of multi-agent systems, to discuss and debate the major issues, and to showcase the latest systems using agent based technology. It will promote a forum for discussion on how agent-based techniques, methods, and tools help system designers to accomplish the mapping between available agent technology and application needs. Other stakeholders should be rewarded with a better understanding of the potential and challenges of the agent-oriented approach.

The conference is organized by the Bioinformatics, Intelligent System and Educational Technology Research Group (http://bisite.usal.es/) of the University of Salamanca. This special issue is based on selected, expanded and significantly revised versions of the best papers presented at the conference:

1. E-nose systems are becoming increasingly important instruments across all industries, especially the fields of food and beverages and biomedicine. Given the inaccurate, unsafe and unreliable dependency on the human nose to detect smells that are highly risky and hazardous to human health, e-nose systems offer a tremendous advantage. E-noses are convenient, highly efficient and can be used in real life to detect various types of odors. In the first paper, De Paz et al. present a virtual organization of agents that integrates different classification techniques and neural networks to perform information fusion from parameters retrieved by E-nose systems. The integral brain in enoses is the data processing system, which classifies odors that have been detected by the detection part of its system. The system mimics how a human brain classifies odors.

2. Roda et al. propose a Multi-Agent architecture (MAS) for healthcare AmI systems. It contributes to treat people with Acquired Brain Injury (ABI) by using specific devices to control the patient's movements and some physiological responses, such as the variation of the heart rate, during her rehabilitation process. In this way, the natural relationship between AmI and MAS is exploited. Acquired Brain Injury (ABI) is becoming an epidemic problem in our society, especially among older adults, being known as "the Silent Epidemic". People with ABI demand solutions in research that offer them with a relearning process such that they can recover not only their physical skills but also their cognitive abilities.

3. New product portfolio selection is a multi-criteria decision making problem including both qualitative and quantitative criteria. Determining the exact values for these criteria is often difficult or even impossible taking into account uncertainty and complexity associated with new product development projects. Relich et al. propose a new project portfolio selection model that uses a fuzzy weighted average approach for ranking new product projects and artificial neural networks for estimating project performance. New product development projects are evaluated according to criteria related to marketing, project team, project performance, risk, and strategy. The use of neural Networks enables more precise evaluation of project performance criteria and provides additional information in portfolio selection.

4. Bozhkov et al. propose a new approach for feature dimensionality reduction based on Reservoir Computing (Echo State Networks). The method is validated with EEG data to identify the common neural signatures based on which the positive and negative valence of human emotions across multiple subjects can be reliably discriminated. The key step in the proposed approach is the Intrinsic Plasticity (IP) adaptation of the reservoir states. Learning Echo State Networks (ESN) with IP maximizes the entropy of the distribution of reservoir vectors given static data as a fixed input, which is supposed to follow Gaussian distribution. The equilibrium reservoir vector is extracted for each static input vector by iterating updates of the reservoir vector until it converges.

5. Carneiro et al. target the problem of performance in the workplace that, asides from complex is often controversial. While organizations and generally competitive working conditions push workers into increasing performance demands, this does not necessarily correlates positively to productivity. Moreover, existing performance monitoring approaches (electronic or not) are often dreaded by workers since they either threat their privacy or are based on productivity measures, with specific side effects. We present a new approach for the problem of performance monitoring that is not based on productivity measures but on the workers' movements while sitting and on the performance of their interaction with the machine. We show that these features correlate with mental fatigue and provide a distributed architecture for the nonintrusive and transparent collection of this data. The easiness in deploying this architecture, its non-intrusive nature, the potential advantages for better human 
resources management and the fact that it is not based on productivity measures will, in our belief, increase the willingness of both organizations and workers to implement this kind of performance management initiatives.

6. Julián et al. propose an approach of a social emotional model, which allows to extract the social emotion of a group of intelligent entities. The emotional model PAD allows to represent the emotion of an intelligent entity in 3-D space, allowing the representation of different emotional states. The social emotional model presented in this paper uses individual emotions of each one of the entities, which are represented in the emotional space PAD. Using a social emotional model within intelligent entities allows the creation of more real simulations, in which emotional states can influence decision-making. The result of this social emotional mode is represented by a series of examples, which are intended to represent a number of situations in which the emotions of each individual modify the emotion of the group. Moreover, the paper introduces an example which employs the proposed model in order to learn and predict future actions trying to influence in the social emotion of a group of people.

\section{Acknowledgements}

We would like to thank all the contributing authors, as well as the reviewers for their hard and highly valuable work. Their work has helped to contribute to the success of this special issue. Finally, the SURF project associated. We hope the reader will share our joy and find this special issue very useful.

This work has been carried out by the project Intelligent System for integrated and sustainable management of urban fleets (SURF). TIN201565515-C4-3-R. Ministerio de Economía y Competitividad (Spain). Fondos Feder.

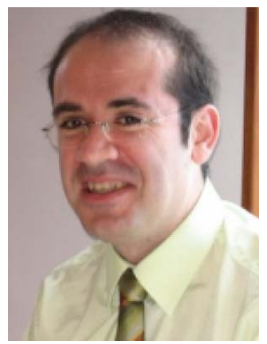

Javier Bajo (PhD). Received a PhD in Computer Science and Artificial Intelligence from the University of Salamanca in 2007. At present he is Tenured Associate Professor at the Universidad Politécnica de Madrid (Spain) and Research Master in Artificial Intelligence (Coordinator). He obtained an Information Technology degree at the University of Valladolid (Spain) in 2001 and an Engineering in Computer Sciences degree at the Pontifical University of Salamanca in 2003. He is co-author of over 300 books, book chapters, journal papers, conference papers published by recognized editorials. He has been member of the organizing and scientific committee of several international symposiums such as CAEPIA, IDEAL, HAIS, KES, IWANN, ACM SAC, IEEE FUSION, PAAMS, etc. and co-author of papers published in recognized journal, workshops and symposiums.

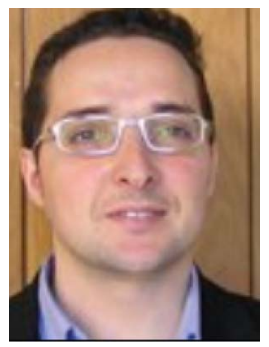

Juan M. Corchado (PhD.). Received a PhD. in Computer Science from the University of Salamanca in 1998 and a PhD. in Artificial Intelligence (AI) from the University of Paisley, Glasgow (UK) in 2000. At present, he is Vice President for Research and Technology Transfer and Full Professor with Chair at the University of Salamanca. He is the Director of the Science Park of the University of Salamanca and Director of the Doctoral School of the University. He has been twice elected Dean at School of Science of the University of Salamanca. Juan M. Corchado is the Director of the BISITE (Bioinformatic, Intelligent Systems and Educational Technology) Research Group, which he created in 2.000, President of the IEEE Systems, Man and Cybernetics Spanish Chapter, Academic Director of the Institute of Digital Art and Animation of the University of Salamanca. He also oversees the Master's programs in Digital Animation, Security, Mobile Telephony, Community Management, Management for TIC enterprises and e-commerce at the University of Salamanca, He is the co-author of over 300 books, book chapters, journal papers, technical reports, etc. published by organizations such as Elsevier, IEEE, IEE, ACM, AaaI, Springer Verlag, Morgan Kaufmann, etc., most of these present practical and theoretical achievements of hybrid AI and distributed systems. He has been President of the organizing and scientific committee of several international symposiums.

Javier Bajo

Artificial Intelligence Department, Polytechnic University of Madrid, Spain E-mail address: jbajo@fi.upm.es

Juan M. Corchado

Computer and Automation Department, University of Salamanca, Spain

E-mail address: corchado@usal.es 